\title{
New York Heart Association Class
}

National Cancer Institute

\section{Source}

National Cancer Institute. New York Heart Association Class. NCI Thesaurus. Code C66909.

A finding associated with a patient based on the functional classification developed by the New York Heart Association (NYHA), for categorizing patients with defined or presumed cardiac disease. The classification system is based on the ability, or lack thereof, to engage in physical activity. The classification system comprises four classes, though NYHA Class III and Class IV are often aggregated for the purpose of outcomes analysis. 